\title{
Physio- und Ergotherapie nach Handverletzungen
}

\author{
Andreas Nusche, Hans-Eberhard Schaller
}

\section{Zusammenfassung}

Die Wiedererlangung ausreichender Sensibilität und Beweglichkeit nach Handverletzungen ist das Ziel jedes handchirurgischen Eingriffs. Eine frühfunktionelle Behandlung ist insbesondere nach Verletzungen der Hand eine wesentliche Voraussetzung zur Vermeidung einer Bewegungseinschränkung. Die Behandlung muss ein reibungsloses Zusammenspiel zwischen Patient, Handchirurg, Ergotherapeut und Physiotherapeut beinhalten. Ein solches Konzept ist in der Regel bei schweren Verletzungen, komplizierten Verläufen und/oder Erkrankungen nur unter stationären Bedingungen in einer entsprechenden Spezialklinik optimal möglich. Kompromisse gehen nicht nur zu Lasten des Verletzten, sondern belasten letztendlich das gesamte soziale Umfeld.

Die Handchirurgie hat sich in den letzten Jahren zu einem wesentlichen Teilgebiet der Chirurgie entwickelt. Damit wird sie der funktionellen, motorischen, sensiblen und sozialen Bedeutung der Hand für den Menschen gerecht.

Neben technischen Möglichkeiten und einer Logistik bis hin zu einem rundum-die-Uhr-Replantationszentrum sind folgende Voraussetzungen für eine erfolgreiche Behandlung der Hand wesentlich:

- rekonstruktive und plastisch chirurgische operative Verfahrensweisen

- Kenntnis der funktionellen Anatomie der Hand

- Teamarbeit mit regelmäßigem Kontakt zwischen Patient, Handchirurg, Physiotherapeut und Ergotherapeut.

OP-JOURNAL 2001; 17: 282-284

(C) Georg Thieme Verlag Stuttgart . New York

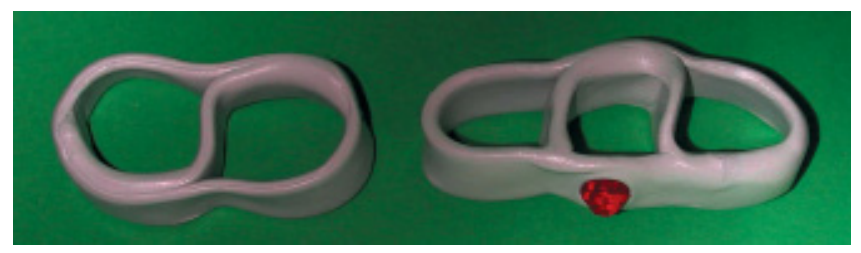

Abb. 1 „8-er“ und „16-er“ Schlingen zur festen Verbindung mehrerer Finger.

Dabei steht der Patient natürlich im Mittelpunkt :

Seine Aufklärung und Bereitschaft zur Kooperation sind die Basis für gute Ergebnisse.

Der Handchirurg überwacht und steuert die postoperative oder ausschließlich konservative Behandlung durch Physiotherapie und Ergotherapie, die sich beide ergänzen.

Neben der Mobilisierung funktioneller Strukturen durch aktive und passive Bewegungsübungen steht die Wiedererlangung der Sensibilität des taktilen Greiforgangs im Vordergrund.

Hilfsmittel, die individuell vom Ergotherapeuten angefertigt werden, können die Mobilisierungen unterstützen.

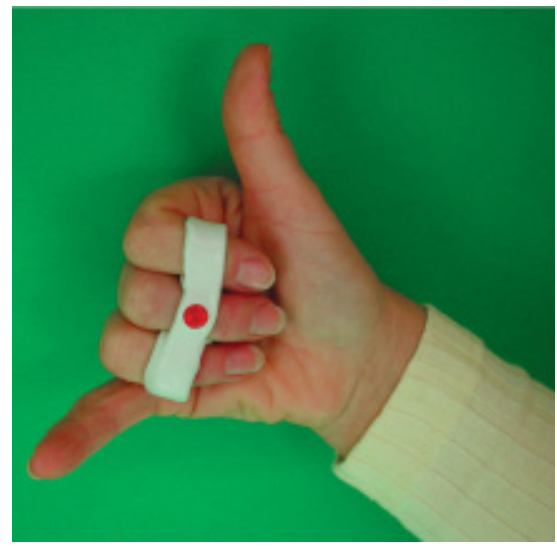

Abb.2 Über die „16-er“ Schlinge nehmen der Zeige-und Ringfinger den Mittelfinger mit.
Die Abb.1 zeigt Mitnehmerschlingen, mit denen mehrere Langfinger verbunden werden können.

Damit nehmen gesunde Nachbarfinger einen bewegungsbeeinträchtigten Finger mit und mobilisieren ihn (Abb.2).

Die Abb. $\mathbf{3}$ zeigt ein sogenanntes „Fingerbrettchen“, welches ebenfalls individuell angefertigt ein Widerlager gegen das pro-

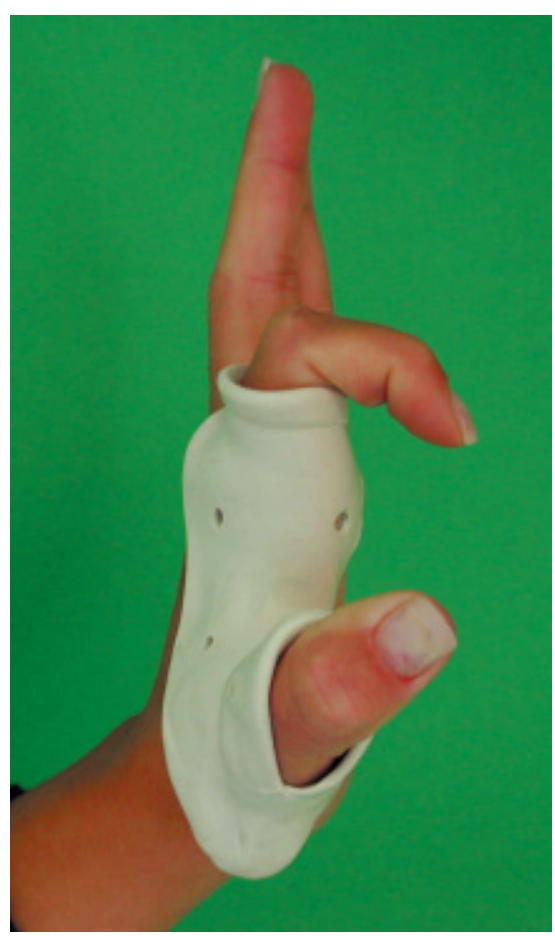

Abb. 3 Das „Fingerbrettchen“ als Hypomochlion zur Verbesserung der Beugung im Mittelgelenk. 

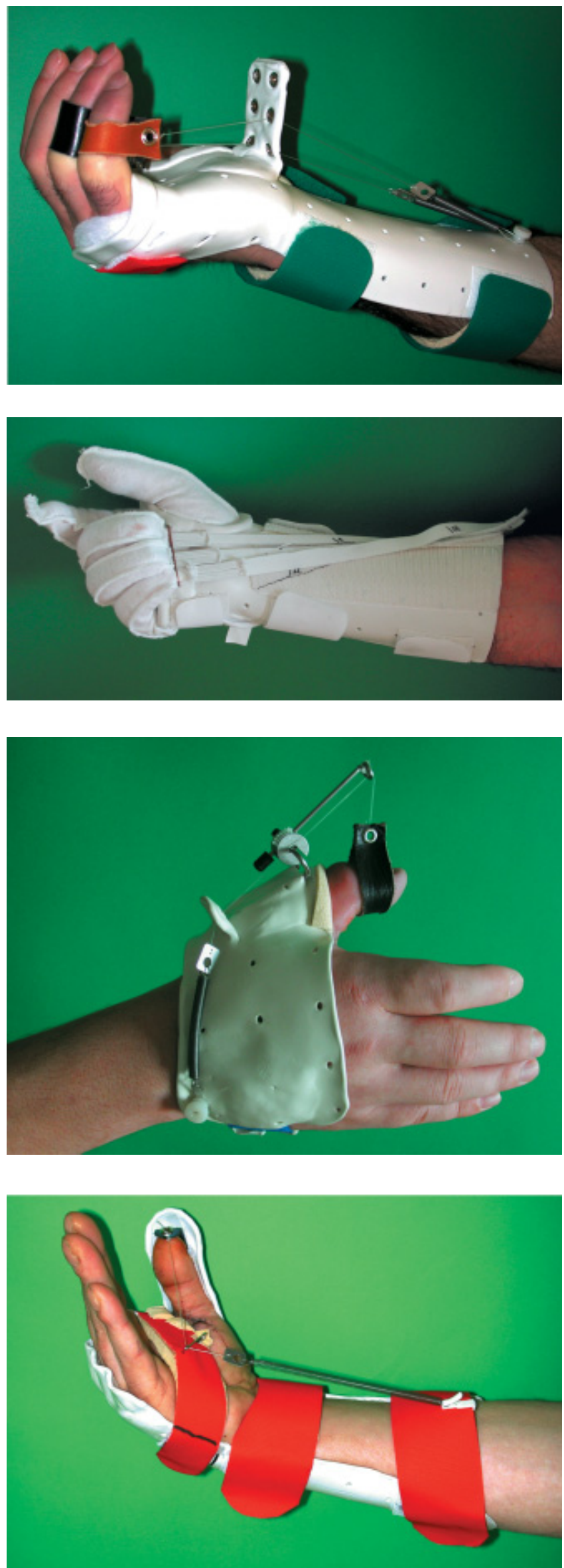

Abb. 4 Beugequengel für das Ring- und Kleinfingermittelgelenk mit Lederschlaufe und Zugfeder.

Abb. 5 Beugehandschuh: Die Fingerkuppen des Handschuhs sind mit Gummibändern verbunden, welche unter Zug an der Polyformschiene befestigt werden. Achte auf den Verlauf der Bänder, die sich alle über dem Scaphoid kreuzen sollen.

Abb. 6 Streckquengel für das Daumenendgelenk. Das Widerlager der Polyformschiene soll knapp proximal des zu quengelnden Gelenkes enden, das Ende muss gut gepolstert werden, um Druckstellen zu vermeiden.

Abb. 7 KleinertSchiene nach Durchtrennung und Naht der Daumenbeugesehne („Daumenkleinert“). ximale Fingerglied bewirkt und damit die Beugung des distal gelegenen Fingergelenkes verstärkt, weil ein Ausweichen des proximalen Fingergliedes verhindert wird.

Häufig müssen die Hilfsmittel während der Behandlung geändert werden, wenn sich zum Beispiel die initiale Schwellung einer Gliedmaße durch Heilung, konsequente Hochlagerung, Eisanwendung oder Lymphdrainage zurückbildet.

Üblich ist auch ein Wechsel zwischen verschiedenen Hilfsmitteln während einer Behandlungsphase. So kann das distal einer Fraktur gelegene Gelenk relativ frühzeitig mit einem Fingerbrettchen beübt werden. Bei weiterer knöcherner Konsolidierung ist ein Wechsel zur Mitnehmerschlinge funktionell wertvoll und bei persistierender Bewegungseinschränkung nach weitgehend abgeschlossener knöcherner Konsolidierung kommt die Quengeltherapie zum Zuge.

Abb. 4 zeigt einen Beugequengel, wobei unterschiedlich starke Federn das Gelenk in die Beugung quengeln.

Sind mehrere oder sogar alle Fingergelenke in der Beweglichkeit eingeschränkt, wie zum Beispiel nach abgelaufener Algodystrophie (M. Sudeck), so kann ein Beuge-(Quengel-) Handschuh angefertigt werden (Abb.5).

Umgekehrt kann bei eingeschränkter Streckung ein Streckquengel angefertigt werden (Abb. 6).

Die wichtigste Maßnahme zur Vermeidung einer Bewegungseinschränkung ist die frühfunktionelle Behandlung.

Eine röntgenologisch anatomisch ausgeheilte Fraktur ist wertlos, wenn die benachbarten Fingergelenke eingesteift sind!

Dies gilt in gleichem Maße auch für die Durchtrennung einer Beugesehne. Die Behandlung in der Kleinert-Schiene (Abb.7) ist ein wichtiges Beispiel für das notwendige Zusammenspiel zwischen Patient, Handchirurg, Ergotherapeut und Physiotherapeut.

Zur Wiedererlangung einer ausreichenden Sensibilität ist ein spezielles Training bei eintretender Nervenregeneration notwendig. Trainingsmethoden ähnlich wie bei der Erlernung der Blindenschrift, Unterscheidung verschiedener Oberflä- 

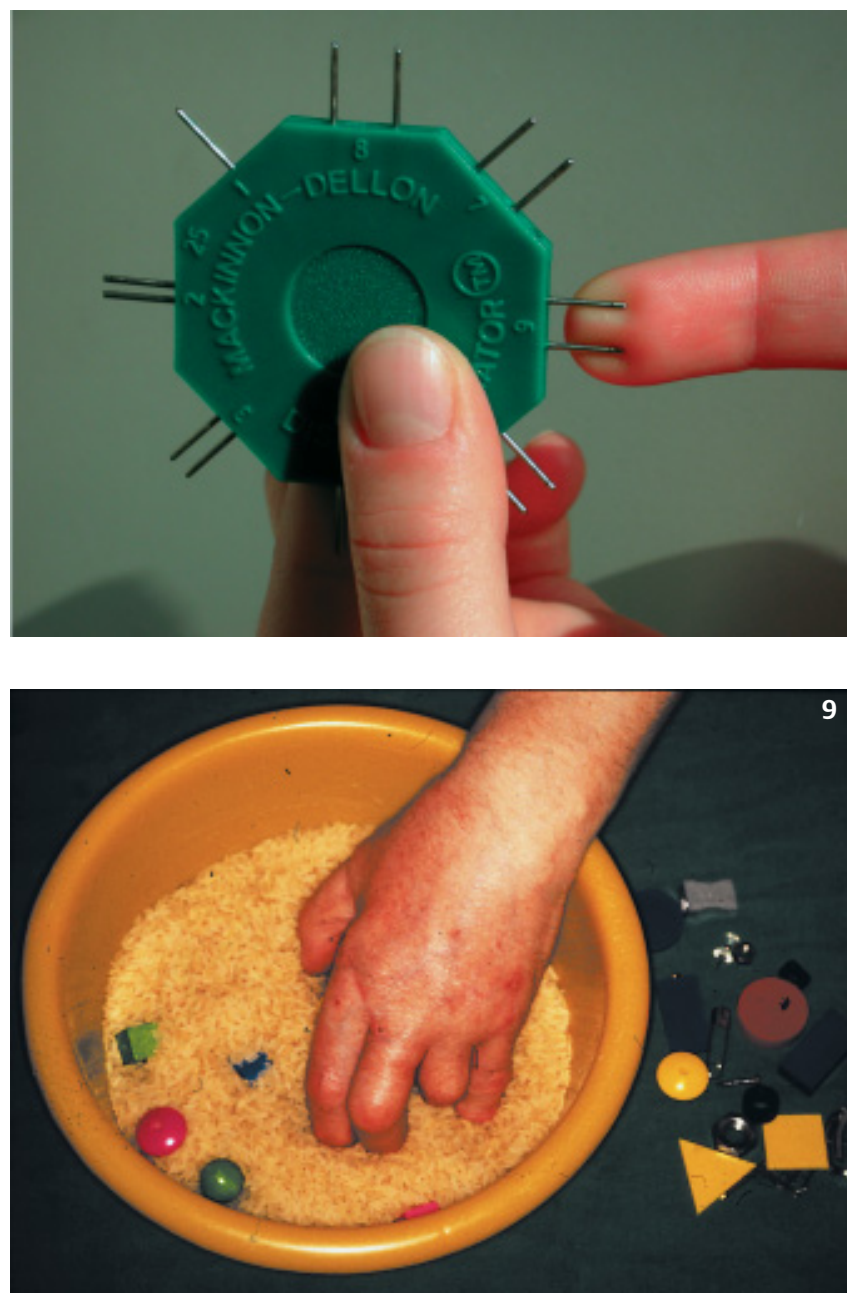

Abb. 9 u.10 Ergotherapeutisches Sensibilitätstraining. chen (rauh, glatt), spitzer und stumpfer Gegenstände (Abb. 8), sowie von Temperaturunterschieden fordern eine individuelle Anpassung des Therapeuten an die Gegebenheiten des Patienten.

Genauso wichtig sind nach erfolgter Wundheilung auch Abhärtungsmaßnahmen (Abb.9u.10) bei vorhersehbaren oder beginnenden Neurombeschwerden.

Die Wiedergewinnung einer angemessenen ausreichenden Handfunktion hängt im Wesentlichen von dem postoperativen Zusammenwirken der verschiedenen Fachdisziplinen ab. Die optimalen Bedingungen bieten hierfür bei schweren Verletzungen oder Erkrankungen speziell eingerichtete Fachabteilungen.

\section{Literatur}

Boscheinen-Morrin, J, Davey, V, Conolly, WB. Physikalische Therapie der Hand. Hippokrates Stuttgart 1988

Dr. med. Andreas Nusche

Assistenzarzt

Prof. Dr. med. Hans-Eberhard Schaller Universitätsprofessor

BG-Unfallklinik Tübingen

Klinik für Hand-, Plastische-, Rekonstruktive und Verbrennungschirurgie an der Eberhard Karls Universität Tübingen Schnarrenbergstr. 95 D-72076 Tübingen

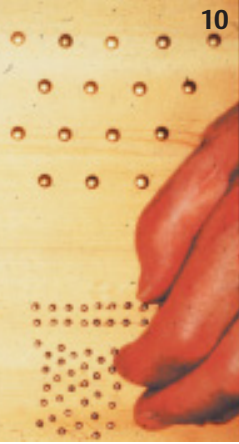

